\title{
High-Pressure Structural and Equation of State Study of Xenotime
}

\author{
Nancy L. Ross, ${ }^{1}$ Jing Zhao, ${ }^{1}$ Lynn A. Boatner, ${ }^{2}$ \\ ${ }^{[1]}$ Department of Geosciences, Virginia Tech, Blacksburg, VA 24060, USA \\ ${ }^{[2]}$ Materials Science and Technology Division, Oak Ridge National Laboratory, Oak Ridge, TN 37831, \\ USA
}

Xenotime has a wide range of formation temperatures in geological environments and is one of the most valuable minerals used for U-Pb geochronology. A major component of xenotime is $\mathrm{YPO}_{4}$ which crystallizes in space group I4 $4_{1}$ /amd (no. 141). The crystal structure of $\mathrm{YPO}_{4}$ is composed of edge-sharing $\mathrm{YO}_{8}$ dodecahedra and $\mathrm{PO}_{4}$ tetrahedra that form chains running parallel to [001]. We studied the effect of pressure on the structure of YPO4 to $9.1 \mathrm{GPa}$ using single-crystal X-ray diffraction. The structure is stable throughout the pressure range studied. Axial compressibilities are anisotropic with [100] approximately 2.4 times more compressible than [001]. Volume reduction of the structure is accommodated by bond length shortening in both the $\mathrm{PO}_{4}$ tetrahedra and $\mathrm{YO}_{8}$ dodecahedra. Interestingly, the shorter Y-O bond lengths show a greater decrease with pressure than the longer $\mathrm{Y}-\mathrm{O}$ bond lengths. A 3rd-order Birch-Murnaghan equation of state fit to the P-V data yielded $\mathrm{K}=143.4(7) \mathrm{GPa}$ with $\mathrm{dK} / \mathrm{dP}=7.0(2)$.

The results from this study are compared with high-pressure studies of other phosphates isostructural with xenotime as well as phosphates isostructural with monazite. Monazite, with ideal formula, $\mathrm{CePO}_{4}$, is monoclinic and crystallizes in space group $\mathrm{P} 2_{1} / n$ (no. 14) with $\mathrm{PO}_{4}$ tetrahedra cross-linked by rare-earth cations in 9-fold coordination with oxygen. Whereas xenotime-type phosphates are known to accommodate smaller rare-earth cations ( $\mathrm{Tb}$ to $\mathrm{Lu}$ ), monazite-type phosphates accommodate larger rare-earth cations ( $\mathrm{Ce}$ to $\mathrm{Gd})$. The effect of cation size on the equations of state of these phosphates will be discussed. 